\title{
Necessary and Sufficient Constraint Qualifications for Solvability of Systems of Infinite Convex Inequalities
}

\author{
M. A. Goberna*, V. Jeyakumar ${ }^{\dagger}$ and M. A. López
}

Revised Version: November 9, 2006

\begin{abstract}
In this paper we present constraint qualifications which completely characterize the Farkas-Minkowski and the locally Farkas-Minkowski convex (linear) inequality systems posed in topological vector spaces. The number of constraints and the dimension of the linear space are arbitrary (possibly infinite). The constraint qualifications considered in this paper are expressed in terms of the solvability of certain parametric convex (linear) systems and the uniform strong duality or the uniform min-max duality relative to the Lagrange (Haar) dual problems of suitable convex (linear) parametric optimization problems.
\end{abstract}

\section{Introduction}

We consider given a convex inequality system

$$
\sigma:=\left\{f_{t}(x) \leq 0, t \in T, x \in C\right\}
$$

where $X$ is a locally convex Hausdorff topological vector space, $C \subset X$ is a closed convex set, $T$ is an arbitrary (possibly infinite) index set, and $f_{t}: X \rightarrow \mathbb{R} \cup\{+\infty\}$ are lower semicontinuous (lsc) proper convex functions for all $t \in T$. We assume throughout the paper the following general assumption

$$
(\mathrm{H}): \quad\left\{\begin{array}{l}
\sigma \text { is solvable, with solution set } A \neq \emptyset, \text { and } \\
\text { each } f_{t} \text { is continuous at least at one point of } A .
\end{array}\right.
$$

We also consider the particular case of linear systems, for which $C=X$ and all the constraint functions $f_{t}$ are affine. Observe that such a linear system

$$
\sigma:=\left\{a_{t}(x) \leq b_{t}, t \in T\right\}
$$

\footnotetext{
${ }^{*}$ Department of Statistics and Operations Research, University of Alicante, Spain. Research supported by DGES and FEDER, Grant MTM2005-08572-C03-01

${ }^{\dagger}$ Department of Applied Mathematics, University of New South Wales, Sydney 2052, Australia.

${ }^{\ddagger}$ Department of Statistics and Operations Research, University of Alicante, Spain. Research supported by DGES and FEDER, Grant MTM2005-08572-C03-01
} 
with $a_{t}: X \rightarrow \mathbb{R}$ linear and $b_{t} \in \mathbb{R}$, for all $t \in T$, satisfies (H) if and only if $A \neq \emptyset$ and $\left\{a_{t}, t \in T\right\}$ is a subset of the continuous dual space $X^{*}$.

In this infinite-dimensional setting, two new constraint qualifications (CQs) for $\sigma$ were recently analyzed in [5] under the names of Farkas-Minkowski and locally FarkasMinkowski. These geometric CQs, that are inspired in the corresponding concepts in linear semi-infinite programming ([6, Notes to Chap. 5]), are completely characterized in this paper in terms of the solvability of the system

$$
f(x)<0, \quad f_{t}(x) \leq 0, \quad t \in T, \quad x \in C,
$$

and the duality behavior of the problem

$$
\begin{aligned}
& \inf f(x) \\
& \text { s.t. } x \in C, \quad f_{t}(x) \leq 0, t \in T,
\end{aligned}
$$

when $f$ ranges over a certain class of convex functions. Obviously, the unsolvability of (1.2) for a certain $f$ is equivalent to assert that the inequality $f(x) \geq 0$ is consequence of $\sigma$.

Observe that $\sigma$ can be reformulated as $\left\{g(x) \in-\mathbb{R}_{+}^{T} ; x \in C\right\}$, where $g: X \rightarrow \mathbb{R}^{T}$ is the function $x \mapsto g(x)$ defined by

$$
g(x)(t)=f_{t}(x), \text { for all } t \in T,
$$

for $x \in X$. Then $g$ is $\mathbb{R}_{+}^{T}$-convex in the sense that, given $x, y \in X$ and $\mu \in[0,1]$,

$$
g(\mu x+(1-\mu) y)-\mu g(x)-(1-\mu) g(y) \in-\mathbb{R}_{+}^{T},
$$

but it is not necessarily continuous. Nevertheless, given $\lambda \in \mathbb{R}_{+}^{(T)}$ (the dual cone of $\mathbb{R}_{+}^{T}$ ), the composition function $\lambda g: X \rightarrow \mathbb{R} \cup\{+\infty\}$ such that $\lambda g=\sum_{t \in T} \lambda_{t} f_{t}$ is a proper lsc convex function.

A new closed cone CQ has been introduced in [10] for systems of the form $\{g(x) \in$ $-S ; x \in C$, where $g: X \rightarrow Z, X$ and $Z$ are Banach spaces, $S$ is a closed convex cone in $Z$ and $g$ is $S$-convex and continuous. From the existence theorem and the Farkas lemma for such kind of systems, the authors derive Lagrangian duality results and optimality conditions for the minimization of convex continuous functions on the solution set of a system satisfying the closed cone CQ. In the same context, the closed cone CQ has been reformulated recently in [1] in order to analyze its connection with different types of duality.

The first objective of this paper is the characterization of the Farkas-Minkowski and the locally Farkas-Minkowski systems through suitable families of consequent inequalities. Concerning $(\mathrm{P})$, it can be seen as a parametric optimization problem with parameter $f$. Hence, given a particular function $f$, the corresponding problem $(\mathrm{P})$, and an associated dual problem, we say that the strong duality holds if both problems have the same optimal value and, moreover, the dual problem is solvable when the optimal value is finite, whereas the min-max duality holds if the solvabilty of the primal problem implies the solvabilty of the dual one with a zero duality gap. In [7], a 
convex system $\sigma$ posed in a finite dimensional space is said to provide uniform duality if the duality gap for the Lagrangian dual problem is zero for all continuous function $f$. In the same vein, we say that $\sigma$ provides uniform strong duality (uniform min-max duality, respectively), relative to a certain dual problem and a certain family of convex functions, when the strong duality (the min-max duality) holds for each particular instance of $(\mathrm{P})$. Thus, the second objective of the paper consists of characterizing the Farkas-Minkowski (locally Farkas-Minkowski) systems as those systems providing uniform strong duality (uniform min-max duality, respectively) for a suitable dual problem (the Lagrange dual problem in the convex case and the Haar's dual in the linear one) and for a suitable class of objective functions.

The layout of the paper is as follows. Section 3 shows that some relaxations of the existence theorem and the Farkas lemma in [10] are still valid for our system $\sigma$, so that is is possible to adapt the main results of [1] to our context. From these results, we caracterize, in Section 4, the Farkas-Minkowski CQ in terms of the uniform strong duality of suitable dual problems as well as in terms of the solvability of the system (1.2). Finally, Section 5 provides a characterization of the locally Farkas-Minkowski $\mathrm{CQ}$ in terms of the uniform min-max duality of suitable dual problems.

\section{Preliminaries}

Let $X$ be a locally convex Hausdorff topological vector space. Let $C$ be a nonempty subset of $X$. We denote by $\mathrm{cl} C$ and $\operatorname{int} C$, respectively, the closure and the interior of $C$. Given a set $D \subset X^{*}$ (or $D \subset X^{*} \times \mathbb{R}$ ), the notation $\operatorname{cl} D$ will stand for the weak*-closure of $D$. The indicator and the support functions of $C$ are denoted respectively by $\delta_{C}$ and $\delta_{C}^{*}$. Let $h: X \rightarrow \mathbb{R} \cup\{+\infty\}$ be a proper convex function. The domain and the epigraph of $h$ are defined as

$$
\operatorname{dom} h:=\{x \in X \mid h(x)<+\infty\} \text { and epi } h:=\{(x, r) \in X \times \mathbb{R} \mid h(x) \leq r\} .
$$

The conjugate function of $h, h^{*}: X^{*} \rightarrow \mathbb{R} \cup\{+\infty\}$, is defined by

$$
h^{*}(v):=\sup \{v(x)-h(x) \mid x \in \operatorname{dom} h\} .
$$

For proper convex lsc functions $g, h: X \rightarrow \mathbb{R} \cup\{+\infty\}$, the infimal convolution of $g$ with $h$, denoted by $g \square h$, is defined by

$$
(g \square h)(x):=\inf _{x_{1}+x_{2}=x}\left\{g\left(x_{1}\right)+h\left(x_{2}\right)\right\},
$$

and the subdifferential of $h$ at $x \in \operatorname{dom} h, \partial h(x)$, is defined as

$$
\partial h(x):=\left\{v \in X^{*} \mid h(y) \geq h(x)+v(y-x) \forall y \in X\right\} .
$$

For a closed and convex set $F \subset X$ and $x \in F, \partial \delta_{F}(x)=N_{F}(x)$, which is known as the normal cone of $F$ at $x$. 
Let $g, h$ and $g_{i}, i \in I$ (where $I$ an arbitrary index set) be lsc and proper convex functions. It is well known from the dual operation that if $\operatorname{dom} g \cap \operatorname{dom} h \neq \emptyset$, then

$$
(g \square h)^{*}=g^{*}+h^{*},(g+h)^{*}=\operatorname{cl}\left(g^{*} \square h^{*}\right) ;
$$

and, if $\sup _{i \in I} g_{i}$ is proper, then

$$
\left(\sup _{i \in I} g_{i}\right)^{*}=\operatorname{cl} \operatorname{co}\left(\inf _{i \in I} g_{i}^{*}\right) .
$$

(See, for instance, [12, 13, Chapter 6]). Clearly, (2.1) and (2.2) imply that

$$
\operatorname{epi}(g+h)^{*}=\operatorname{cl}\left(\operatorname{epi} g^{*}+\operatorname{epi} h^{*}\right) \text { and } \operatorname{epi}\left(\sup _{i \in I} g_{i}\right)^{*}=\operatorname{cl} \operatorname{co}\left(\cup_{i \in I} \operatorname{epi} g_{i}^{*}\right) .
$$

The closure operation in the first equation is superfluous if one of $g$ and $h$ is continuous at some $a \in \operatorname{dom} g \cap \operatorname{dom} h$ ([5, Lemma 1]). Then, since epi $g^{*}+$ epi $^{*}$ is weak*-closed,

$$
\partial(g+h)(x)=\partial g(x)+\partial h(x), \forall x \in \operatorname{dom} g \cap \operatorname{dom} h .
$$

(See [2, Theorem 3.1].)

If $F$ is a closed convex set in $X$, and $h$ is continuous at some point of $a \in F \cap \operatorname{dom} h$, then epi $h^{*}+\operatorname{epi} \delta_{F}^{*}$ is weak ${ }^{*}$-closed and

$$
\bar{x} \in F \cap \operatorname{dom} h \text { minimizes } h \text { on } F \Leftrightarrow 0 \in \partial h(\bar{x})+N_{F}(\bar{x}) .
$$

([2, Theorem 4.1]).

We denote by $\mathbb{R}_{+}^{(T)}$ the positive cone in $\mathbb{R}^{(T)}$, the so-called space of generalized finite sequences $\lambda=\left(\lambda_{t}\right)_{t \in T}$ such that $\lambda_{t} \in \mathbb{R}$, for each $t \in T$, and with only finitely many $\lambda_{t}$ different from zero.

The characteristic cone of $\sigma$ is the convex cone, $K$, generated by the union of the closed convex sets epi $f_{t}^{*}, t \in T$, and the closed convex cone epi $\delta_{C}^{*}$, i.e.

$$
K:=\operatorname{cone}\left\{\bigcup_{t \in T} \operatorname{epi} f_{t}^{*}\right\}+\operatorname{epi} \delta_{C}^{*} .
$$

By a well-known version of Farkas Lemma ([4], [9]), given $v \in X^{*}$ and $\rho \in \mathbb{R}$,

$$
v(x) \leq \rho \text { is a consequence of } \sigma \Leftrightarrow(v, \rho) \in \operatorname{cl} K .
$$

\section{Solvability of $S$-convex systems revisited}

Let $X$ and $C$ be as in Section 1 . Let $Z$ be a locally convex Hausdorff topological vector space and let $S$ be a closed convex cone in $Z$, with dual cone

$$
S^{+}:=\left\{\lambda \in Z^{*} \mid \lambda(z) \geq 0 \forall z \in S\right\} .
$$

Let $g: X \rightarrow Z$ be a $S$-convex mapping; i.e., for all $x, y \in X$ and every $\mu \in[0,1]$

$$
g(\mu x+(1-\mu) y)-\mu g(x)-(1-\mu) g(y) \in-S .
$$

As a consequence of (3.1), the composed function $\lambda g: X \rightarrow \mathbb{R}$ is convex for all $\lambda \in S^{+}$. The next result is a relaxed versions of [10, Lemma 2.1]. 
Lemma 3.1 If $\lambda g$ is lsc for all $\lambda \in S^{+}$, then the following statements hold:

(i) $C \cap g^{-1}(-S) \neq \emptyset$ if and only if

$$
(0,-1) \notin \mathrm{cl}\left(\bigcup_{\lambda \in S^{+}} \operatorname{epi}(\lambda g)^{*}+\operatorname{epi} \delta_{C}^{*}\right) .
$$

(ii) If $C \cap g^{-1}(-S) \neq \emptyset$, then

$$
\operatorname{epi}_{C \cap g^{-1}(-S)}^{*}=\mathrm{cl}\left(\bigcup_{\lambda \in S^{+}} \operatorname{epi}(\lambda g)^{*}+\operatorname{epi} \delta_{C}^{*}\right) .
$$

Proof. The same argument of [10, Lemma 2.1] applies, just observing that the continuity of $g$ can be relaxed to the lsc property of the composite functions $\lambda g$ for all $\lambda \in S^{+}$.

Definition 3.1 The consistent $S$-convex system $\{g(x) \in-S ; x \in C\}$ satisfies the closed cone constraint qualification (CCCQ) if

$$
\bigcup_{\lambda \in S^{+}} \operatorname{epi}(\lambda g)^{*}+\operatorname{epi} \delta_{C}^{*}
$$

is weak*-closed.

As a consequence of Lemma 3.1(ii), if $\{g(x) \in-S ; x \in C\}$ is consistent and $\lambda g$ is lsc for all $\lambda \in S^{+}$, then it satisfies (CCCQ) if and only if the alternative form of (CCCQ),

$$
\operatorname{epi}_{C \cap g^{-1}(-S)}^{*} \subset \bigcup_{\lambda \in S^{+}} \operatorname{epi}(\lambda g)^{*}+\operatorname{epi} \delta_{C}^{*}
$$

given in [1], holds.

\section{Characterizing the Farkas-Minkowski systems}

Definition 4.1 We say that the system $\sigma$ in (1.1) satisfying (H) is Farkas-Minkowski (FM, in brief) if $K$ is weak ${ }^{*}$-closed.

First we show that $\sigma$ is FM if and only if (CCCQ) holds although here $X$ is not necessarily a Banach space and $Z:=\mathbb{R}^{T}$ is not even normable when $T$ is infinite. Recall that, in our setting, $g$ is the mapping introduced in (1.3), $S=\mathbb{R}_{+}^{T}$ (which is closed and convex) and $S^{+}=\mathbb{R}_{+}^{(T)}$.

Given $\lambda \in \mathbb{R}_{+}^{(T)}$, if $\sum_{t \in T} \lambda_{t}>0$, by (2.3) and assumption $(\mathrm{H})$, we have

$$
\operatorname{epi}(\lambda g)^{*}=\operatorname{cl}\left(\sum_{t \in T} \operatorname{epi}\left(\lambda_{t} f_{t}\right)^{*}\right)=\operatorname{cl}\left(\sum_{t \in T} \lambda_{t} \operatorname{epi} f_{t}^{*}\right)=\sum_{t \in T} \lambda_{t} \operatorname{epi} f_{t}^{*}
$$


whereas epi $(0 g)^{*}=\mathbb{R}_{+}(0,1)$ by definition. Then,

$$
\bigcup_{\lambda \in S^{+}} \operatorname{epi}(\lambda g)^{*}=\operatorname{cone}\left(\bigcup_{t \in T} \operatorname{epi} f_{t}^{*} \cup\{(0,1)\}\right)
$$

and, observing that $(0,1) \in \operatorname{epi} \delta_{C}^{*}$ (because $\delta_{C}^{*}$ is an homogeneous function), we get

$$
K=\operatorname{cone}\left(\bigcup_{\lambda \in S^{+}} \operatorname{epi}(\lambda g)^{*}+\operatorname{epi} \delta_{C}^{*}\right)=\bigcup_{\lambda \in S^{+}} \operatorname{epi}(\lambda g)^{*}+\operatorname{epi} \delta_{C}^{*} .
$$

The next result is the version, in our context, of [1, Lemma 3.1].

Lemma $4.1 \sigma$ is FM if and only if

$$
\operatorname{epi}_{A}^{*} \subset \bigcup_{\lambda \in S^{+}} \operatorname{epi}\left(\lambda g+\delta_{C}\right)^{*}
$$

Proof. According to the characterization of the FM condition in (3.2), it is enough to prove that $K=\bigcup_{\lambda \in S^{+}} \operatorname{epi}\left(\lambda g+\delta_{C}\right)^{*}$. In fact, given $\lambda \in S^{+}$, again by (2.3) and assumption $(\mathrm{H})$, we obtain

$$
\begin{aligned}
\operatorname{epi}\left(\lambda g+\delta_{C}\right)^{*} & =\operatorname{epi}\left(\sum_{t \in T} \lambda_{t} f_{t}+\delta_{C}\right)^{*}=\operatorname{cl}\left(\sum_{t \in T} \lambda_{t} \text { epi } f_{t}^{*}+\operatorname{epi} \delta_{C}^{*}\right) \\
& =\sum_{t \in T} \lambda_{t} \text { epi } f_{t}^{*}+\operatorname{epi} \delta_{C}^{*}=\operatorname{epi}(\lambda g)^{*}+\operatorname{epi} \delta_{C}^{*} .
\end{aligned}
$$

Thus,

$$
K=\bigcup_{\lambda \in S^{+}} \operatorname{epi}(\lambda g)^{*}+\operatorname{epi} \delta_{C}^{*}=\bigcup_{\lambda \in S^{+}}\left(\operatorname{epi}(\lambda g)^{*}+\operatorname{epi} \delta_{C}^{*}\right)=\bigcup_{\lambda \in S^{+}} \operatorname{epi}\left(\lambda g+\delta_{C}\right)^{*} .
$$

Observe that assumption $(\mathrm{H})$ does not entail the continuity of $\delta_{A}$ at any point of $A$, since $A$ can be a singleton (consider, for instance, $X=C=\mathbb{R}$, and $f_{t}(x)=t|x|, t \in$ $\left[1,+\infty[)\right.$. Nevertheless, $\delta_{A}$ is lsc, proper and convex, and these properties guarantee a good behavior of $\sigma$ in the context of the Fenchel duality.

Recall that the Fenchel dual problem of $(\mathrm{P})$ is

$$
\left(\mathrm{D}_{\mathrm{F}}\right) \quad \sup _{u \in X^{*}}\left\{-f^{*}(u)-\delta_{A}^{*}(-u)\right\} .
$$

Lemma 4.2 [3] Let $f: X \rightarrow \mathbb{R} \cup\{+\infty\}$ be a lsc proper convex function with $A \cap$ $\operatorname{dom} f \neq \emptyset$. If epi $f^{*}+\operatorname{epi}_{A}^{*}$ is weak closed then the strong duality holds for $(\mathrm{P})-\left(\mathrm{D}_{\mathrm{F}}\right)$, i.e.,

$$
\inf _{x \in A} f(x)=\max _{u \in X^{*}}\left\{-f^{*}(u)-\delta_{A}^{*}(-u)\right\} .
$$


The next result characterizes the FM property in terms of the strong duality of the Lagrange dual problem associated with (P), denoted by (D) and defined by

$$
\text { (D) } \sup _{\lambda \in \mathbb{R}_{+}^{(T)}} \inf _{x \in C}\left\{f(x)+\sum_{t \in T} \lambda_{t} f_{t}(x)\right\} .
$$

It is easy to show the weak dual inequality:

$$
\sup _{\lambda \in \mathbb{R}_{+}^{(T)}} \inf _{x \in C}\left\{f(x)+\sum_{t \in T} \lambda_{t} f_{t}(x)\right\} \leq \inf _{x \in A} f(x) .
$$

Theorem 4.1 Suppose that the system $\sigma$ in (1.1) satisfies $(\mathrm{H})$. Then, the following statements are equivalent:

(i) $\sigma$ is FM.

(ii) For every lsc proper convex function $f: X \rightarrow \mathbb{R} \cup\{+\infty\}$ with $A \cap \operatorname{dom} f \neq \emptyset$ and epi $f^{*}+\operatorname{epi}_{A}^{*}$ weak ${ }^{*}$ closed, the strong duality holds, i.e.

$$
\inf _{x \in A} f(x)=\max _{\lambda \in \mathbb{R}_{+}^{(T)}} \inf _{x \in C}\left\{f(x)+\sum_{t \in T} \lambda_{t} f_{t}(x)\right\} .
$$

(iii) For every $v \in X^{*}$

$$
\inf _{x \in A} v(x)=\max _{\lambda \in \mathbb{R}_{+}^{(T)}} \inf _{x \in C}\left\{v(x)+\sum_{t \in T} \lambda_{t} f_{t}(x)\right\} .
$$

Proof. The argument to prove the equivalence $(\mathrm{i}) \Leftrightarrow($ iii) is the same as in $[1$, Theorem 3.2], and Lemma 4.1 is used.

(ii) $\Rightarrow$ (iii) is trivial, and (iii) $\Rightarrow$ (ii) can be proved in a similar way as $[1$, Theorem 4.3]. For illustrative purposes we include here the proof.

According to Lemma 4.2, there exists $\bar{u} \in X^{*}$ such that

$$
\begin{aligned}
\inf _{x \in A} f(x) & =-f^{*}(\bar{u})-\delta_{A}^{*}(-\bar{u}) \\
& =-f^{*}(\bar{u})+\inf _{x \in A} \bar{u}(x) .
\end{aligned}
$$

As (iii) holds, there also exists $\bar{\lambda} \in \mathbb{R}_{+}^{(T)}$ verifying

$$
\begin{aligned}
\inf _{x \in A} f(x) & =-f^{*}(\bar{u})+\inf _{x \in C}\left\{\bar{u}(x)+\sum_{t \in T} \bar{\lambda}_{t} f_{t}(x)\right\} \\
& =\inf _{x \in C}\left\{\bar{u}(x)-f^{*}(\bar{u})+\sum_{t \in T} \bar{\lambda}_{t} f_{t}(x)\right\} \\
& \leq \inf _{x \in C}\left\{f(x)+\sum_{t \in T} \bar{\lambda}_{t} f_{t}(x)\right\},
\end{aligned}
$$

and this, together with (4.1), yields (ii). 
The following characterization of the FM constraint qualification is a straightforward consequence of Theorem 4.1.

Corollary 4.1 Suppose that the system $\sigma$ in (1.1) satisfies (H). Then, the following statements are equivalent:

(i) $\sigma$ is FM.

(ii) For every lsc proper convex function $f: X \rightarrow \mathbb{R} \cup\{+\infty\}$ with $A \cap \operatorname{dom} f \neq \emptyset$ and epi $f^{*}+\operatorname{epi} \delta_{A}^{*}$ weak $k^{*}$ closed, the inequality $f(x) \geq 0$ is a consequence of $\sigma$ if and only if

$$
\left(\exists \lambda \in \mathbb{R}_{+}^{(T)}\right)(\forall x \in C) f(x)+\sum_{t \in T} \lambda_{t} f_{t}(x) \geq 0 .
$$

(iii) For every $v \in X^{*}$ and every $\gamma \in \mathbb{R}$, the affine inequality $v(x) \geq \gamma$ is a consequence of $\sigma$ if and only if

$$
\left(\exists \lambda \in \mathbb{R}_{+}^{(T)}\right)(\forall x \in C) v(x)+\sum_{t \in T} \lambda_{t} f_{t}(x) \geq \gamma .
$$

Consider now the following particular case of $(\mathrm{P})$ :

$$
\begin{array}{ll}
(\mathrm{LOP}) & \inf v(x) \\
& \text { s.t. } x \in X, a_{t}(x) \geq b_{t}, t \in T,
\end{array}
$$

where $\left\{v ; a_{t}, t \in T\right\} \subset X^{*}$ and $\left\{b_{t}, t \in T\right\} \subset \mathbb{R}$.

(LOP) is an ordinary (an infinite) linear programming problem when the dimension of $X$ and the set $T$ are finite (infinite, respectively), and it is a linear semi-infinite programming problem when either the dimension of $X$ or the set $T$ (but not both) is finite. The next result characterizes the FM property of $\sigma=\left\{a_{t}(x) \geq b_{t}, t \in T\right\}$ in terms of the strong duality between (LOP) and the so-called Haar's dual problem

$$
\begin{aligned}
& (\widetilde{\mathrm{D}}) \quad \sup \sum_{t \in T} \lambda_{t} b_{t}, \\
& \text { s.t. } \lambda \in \mathbb{R}_{+}^{(T)}, \quad \sum_{t \in T} \lambda_{t} a_{t}=v .
\end{aligned}
$$

The feasible set of $(\widetilde{\mathrm{D}})$ is denoted by $\Lambda$, i.e.

$$
\Lambda=\left\{\lambda \in \mathbb{R}_{+}^{(T)} \mid \sum_{t \in T} \lambda_{t} a_{t}=v\right\} .
$$

The following weak dual inequality for the Haar's dual pair is straightforward

$$
\sup _{\lambda \in \Lambda} \sum_{t \in T} \lambda_{t} b_{t} \leq \inf _{x \in A} v(x) .
$$


Lemma 4.3 In relation to the Haar's dual pair, the following equality holds

$$
\sup _{\lambda \in \mathbb{R}_{+}^{(T)}} \inf _{x \in X}\left\{f(x)+\sum_{t \in T} \lambda_{t} f_{t}(x)\right\}=\sup _{\lambda \in \Lambda} \sum_{t \in T} \lambda_{t} b_{t},
$$

where $f()=.v($.$) and f_{t}()=.b_{t}-a_{t}(),. t \in T$.

Proof. We can write

$$
f(x)+\sum_{t \in T} \lambda_{t} f_{t}(x)=\sum_{t \in T} \lambda_{t} b_{t}+\left(v-\sum_{t \in T} \lambda_{t} a_{t}\right)(x),
$$

and

$$
\lambda \notin \Lambda \Longrightarrow \inf _{x \in X}\left\{f(x)+\sum_{t \in T} \lambda_{t} f_{t}(x)\right\}=-\infty,
$$

because $X$ separates points of $X^{*}$. Then both expressions in (4.7) have the same value, either $-\infty$ when $\Lambda=\emptyset$ (we assume, in $\mathbb{R}$, $\sup \emptyset=-\infty$ ) or greater than $-\infty$ when $\Lambda \neq \emptyset$.

Theorem 4.2 Consider the linear system $\sigma=\left\{a_{t}(x) \geq b_{t}, t \in T\right\}$ satisfying $(\mathrm{H})$. Then, the following statements are equivalent:

(i) $\sigma$ is FM.

(ii) The convex cone in $X^{*} \times \mathbb{R}$

$$
\text { cone }\left\{\left(a_{t}, b_{t}\right), t \in T ;(0,-1)\right\}
$$

is weak $k^{*}$-closed.

(iii) For every $v \in X^{*}$,

$$
\inf _{x \in A} v(x)=\max _{\lambda \in \Lambda} \sum_{t \in T} \lambda_{t} b_{t} .
$$

Proof. $[(i) \Leftrightarrow($ ii $)]$ It comes from the fact that

$$
K=\operatorname{cone}\left\{\bigcup_{t \in T} \operatorname{epi}\left(b_{t}-a_{t}(.)\right)^{*}\right\}+\operatorname{epi} \delta_{X}^{*},
$$

together with

$$
\left(b_{t}-a_{t}(.)\right)^{*}=\delta_{\left\{-a_{t}\right\}}-b_{t}, t \in T, \text { and } \delta_{X}^{*}=\delta_{\{0\}} .
$$

Therefore

$$
\text { cone }\left\{\left(a_{t}, b_{t}\right), t \in T ;(0,-1)\right\}=-K,
$$

and the definition of the FM property applies.

$[(\mathrm{i}) \Leftrightarrow(\mathrm{iii})]$ It is a straightforward consequence of Lemma 4.3 and the equivalence (i) $\Leftrightarrow$ (iii) in Theorem 4.1. 
Example 3.1 Let $X=\mathbb{R}^{2}$ and $\sigma=\left\{x_{1}+t^{2} x_{2} \geq 2 t, t \in\right] 0,+\infty[\}$. It can easily be seen that $A=\left\{x \in \mathbb{R}_{+}^{2} \mid x_{1} x_{2} \geq 1\right\}$. We discuss four possibilities for $v \in X^{*}=\mathbb{R}^{2}$ :

(i) $v \notin \mathbb{R}_{+}^{2}$. Then

$$
\sup _{\lambda \in \Lambda} \sum_{t \in T} \lambda_{t} b_{t}=2 \sum_{t \in T} t \lambda_{t}=\inf _{x \in A} v(x)=-\infty(\text { with } \Lambda=\emptyset) .
$$

(ii) $v \in \mathbb{R}_{++}^{2}$. Then the unique optimal solutions of (LOP) and ( $\left.\widetilde{\mathrm{D}}\right)$ are

$$
\bar{x}=\left(\sqrt{\frac{v_{2}}{v_{1}}}, \sqrt{\frac{v_{1}}{v_{2}}}\right) \text { and } \bar{\lambda}_{t}=\left\{\begin{array}{cc}
v_{1}, & t=\sqrt{\frac{v_{2}}{v_{1}}}, \\
0, & \text { otherwise }
\end{array}, t \in\right] 0,+\infty[\text {, }
$$

with

$$
\sum_{t \in T} \bar{\lambda}_{t} b_{t}=v(\bar{x})=2 \sqrt{v_{1} v_{2}}
$$

(iii) $v_{1} v_{2}=0, v \in \mathbb{R}_{+}^{2} \backslash 0$. Then

$$
\sup _{\lambda \in \Lambda} \sum_{t \in T} \lambda_{t} b_{t}=-\infty<0=\inf _{x \in A} v(x) .
$$

Thus condition (iii) in Theorems 4.1 and 4.2 fails.

(iv) $v=0$. Any point of $A$ is an optimal solution of (LOP) whereas the unique feasible solution of $(\widetilde{\mathrm{D}})$ is $\bar{\lambda}_{t}=0$, for all $t \in T$. Thus,

$$
\max _{\lambda \in \Lambda} \sum_{t \in T} \lambda_{t} b_{t}=\min _{x \in A} v(x)=0 .
$$

\section{Characterizing the locally Farkas-Minkowski systems}

Recently, necessary and sufficient constraint qualifications for a min-max duality were established in [8] for partially finite convex programming problems, where the constraint map $g: X \rightarrow Z$ is affine and $Z=\mathbb{R}^{m}$ and the objective function $f$ is continuous. In this section, we give a $\mathrm{CQ}$ which is necessary and sufficient for the Lagrangian min-max duality

$$
\min _{x \in A} f(x)=\max _{\lambda \in \mathbb{R}_{+}^{(T)}} \inf _{x \in C}\left\{f(x)+\sum_{t \in T} \lambda_{t} f_{t}(x)\right\},
$$

where $f: X \rightarrow \mathbb{R} \cup\{+\infty\}$ is a lsc proper convex function. 
Definition 5.1 We say that the system $\sigma$ in (1.1) satisfying $(\mathrm{H})$ is locally FarkasMinkowski (LFM, in brief) if

$$
N_{A}(x)=N_{C}(x)+\operatorname{cone}\left(\bigcup_{t \in T(x)} \partial f_{t}(x)\right) \text { for all } x \in A,
$$

where

$$
T(x):=\left\{t \in T \mid f_{t}(x)=0\right\}
$$

is the set of active indices at $x$.

It can be proved that if $\sigma$ is FM then $\sigma$ is LFM. This may also follow from the following characterizations of the LFM CQ in terms of the Lagrangian.

Theorem 5.1 Suppose that the system $\sigma$ in (1.1) satisfies (H). Then, the following statements are equivalent:

(i) $\sigma$ is LFM.

(ii) For every lsc proper convex function $f: X \rightarrow \mathbb{R} \cup\{+\infty\}$ that is continuous at least at a point of $A \cap \operatorname{dom} f$ and that attains its infimum value at $A$, the Lagrangian min-max duality holds, i.e.

$$
\min _{x \in A} f(x)=\max _{\lambda \in \mathbb{R}_{+}^{(T)}} \inf _{x \in C}\left\{f(x)+\sum_{t \in T} \lambda_{t} f_{t}(x)\right\} .
$$

(iii) For every $v \in X^{*}$ that attains its infimum value at $A$ one has

$$
\min _{x \in A} v(x)=\max _{\lambda \in \mathbb{R}_{+}^{(T)}} \inf _{x \in C}\left\{v(x)+\sum_{t \in T} \lambda_{t} f_{t}(x)\right\} .
$$

Proof. The assumption (H) on $\sigma$ guarantees that $\partial\left(\sum_{t \in T} \lambda_{t} f_{t}\right)(x)=\sum_{t \in T} \lambda_{t} \partial f_{t}(x)$ for all $\lambda \in \mathbb{R}_{+}^{(T)}$ and for all $x \in A$.

$[(\mathrm{i}) \Rightarrow(\mathrm{ii})]$ Let $f$ be a lsc proper convex function $f: X \rightarrow \mathbb{R} \cup\{+\infty\}$ that is continuous at some point of $A \cap \operatorname{dom} f$, and let $\bar{x} \in A$ be a minimum point, i.e. $f(\bar{x})=\min _{x \in A} f(x)$. Then, by the optimality condition (2.4), there exists $p \in \partial f(\bar{x})$ such that $-p \in N_{A}(\bar{x})$. It follows from (i) that

$$
-p \in N_{C}(\bar{x})+\operatorname{cone}\left(\bigcup_{t \in T(\bar{x})} \partial f_{t}(\bar{x})\right),
$$

and so, there exists $\bar{\lambda} \in \mathbb{R}_{+}^{(T)}$ with $\sum_{t \in T} \bar{\lambda}_{t} f_{t}(\bar{x})=0$ such that

$$
-p \in N_{C}(\bar{x})+\partial\left(\sum_{t \in T} \bar{\lambda}_{t} f_{t}\right)(\bar{x})
$$

This gives rise to

$$
\begin{aligned}
0 & \in \partial f(\bar{x})+\partial\left(\sum_{t \in T} \bar{\lambda}_{t} f_{t}\right)(\bar{x})+N_{C}(\bar{x}) \\
& =\partial\left(f+\sum_{t \in T} \bar{\lambda}_{t} f_{t}\right)(\bar{x})+N_{C}(\bar{x})
\end{aligned}
$$


so that $\bar{x}$ is a minimizer of $f+\sum_{t \in T} \bar{\lambda}_{t} f_{t}$ on $C$, i.e.

$$
\inf _{x \in C}\left\{f(x)+\sum_{t \in T} \bar{\lambda}_{t} f_{t}(x)\right\} \geq f(\bar{x}) .
$$

This inequality and the weak dual inequality (4.1) together yield (ii).

$[($ ii $) \Rightarrow($ iii $)]$. Trivial.

[(iii) $\Rightarrow(\mathrm{i})]$. Assume that (iii) holds and let $x \in A$ be arbitrary. If $u \in N_{A}(x)$ then $-u(x)=\min _{y \in A}-u(y)$. Now, by (iii), there exists $\bar{\lambda} \in \mathbb{R}_{+}^{(T)}$ such that

$$
-u(x)=\min _{y \in C}\left\{-u(y)+\sum_{t \in T} \bar{\lambda}_{t} f_{t}(y)\right\} .
$$

This gives, by taking $y=x$ above, $\sum_{t \in T} \bar{\lambda}_{t} f_{t}(x)=0$ and

$$
\begin{aligned}
u & \in \partial\left(\delta_{C}+\sum_{t \in T} \bar{\lambda}_{t} f_{t}\right)(x)=\partial \delta_{C}(x)+\partial\left(\sum_{t \in T} \bar{\lambda}_{t} f_{t}\right)(x) \\
& =N_{C}(x)+\operatorname{cone}\left(\bigcup_{t \in T(x)} \partial f_{t}(x)\right) .
\end{aligned}
$$

Hence, (i) holds, as the converse inclusion $N_{C}(x)+\operatorname{cone}\left(\bigcup_{t \in T(x)} \partial f_{t}(x)\right) \subset N_{A}(x)$ is always satisfied.

Finally, we give the LFM counterparts of Corollary 4.1 and Theorem 4.2.

Corollary 5.1 Suppose that the system $\sigma$ in (1.1) satisfies (H). Then, the following statements are equivalent:

(i) $\sigma$ is LFM.

(ii) For every lsc proper convex function $f: X \rightarrow \mathbb{R} \cup\{+\infty\}$ that is continuous at least at a point of $A \cap \operatorname{dom} f$ and such that $f(a)=0$ at some $a \in A$, the inequality $f(x) \geq 0$ is a consequence of $\sigma$ if and only if

$$
\left(\exists \lambda \in \mathbb{R}_{+}^{(T)}\right)(\forall x \in C) f(x)+\sum_{t \in T} \lambda_{t} f_{t}(x) \geq 0 .
$$

(iii) For every $v \in X^{*}$ and every $a \in A$, the affine inequality $v(x-a) \geq 0$ is a consequence of $\sigma$ if and only if

$$
\left(\exists \lambda \in \mathbb{R}_{+}^{(T)}\right)(\forall x \in C) v(x)+\sum_{t \in T} \lambda_{t} f_{t}(x) \geq v(a) .
$$

Proof. It is an immediate consequence of Theorem 5.1

Theorem 5.2 Consider the linear system $\sigma=\left\{a_{t}(x) \geq b_{t}, t \in T\right\}$ satisfying $(\mathrm{H})$. Then, the following statements are equivalent:

(i) $\sigma$ is LFM. 
(ii) For every $v \in X^{*}$ and for every $\bar{x} \in A$

$$
v(x) \geq v(\bar{x}) \text { is a consequence of } \sigma \Leftrightarrow v \in \text { cone }\left\{a_{t}, t \in T(\bar{x})\right\} \text {. }
$$

(iii) For every $v \in X^{*}$ that attains its infimum value at $A$ one has

$$
\min _{x \in A} v(x)=\max _{\lambda \in \Lambda} \sum_{t \in T} \lambda_{t} b_{t} .
$$

Proof. [(i) $\Leftrightarrow($ ii) $]$ Since $N_{X}(\bar{x})=0$ and $\partial f_{t}=\left\{-a_{t}\right\}, t \in T, \sigma$ is LFM if and only if $N_{A}(\bar{x})=-$ cone $\left\{a_{t}, t \in T(\bar{x})\right\}$ for all $\bar{x} \in A$. Observing that $-v \in N_{A}(\bar{x})$ if and only if $v(x) \geq v(\bar{x})$ is consequence of $\sigma$, the conclusion is immediate.

[(ii) $\Rightarrow$ (iii)] Let $\bar{x} \in A$ such that $v(\bar{x})=\min _{x \in A} v(x)$. From (ii) we get $v \in \operatorname{cone}\left\{a_{t}, t \in\right.$ $T(\bar{x})\}$. Let $\bar{\lambda} \in \mathbb{R}_{+}^{(T)}$ such that $\sum_{t \in T} \bar{\lambda}_{t}\left(b_{t}-a_{t}(\bar{x})\right)=0$ and $v=\sum_{t \in T} \bar{\lambda}_{t} a_{t}$. Then $v(\bar{x})=\sum_{t \in T} \bar{\lambda}_{t} b_{t}$ and (iii) holds by the weak dual inequality (4.6).

[(iii) $\Rightarrow($ ii $)]$ Let $v \in X^{*}$ and $\bar{x} \in A$ be such that $v(x) \geq v(\bar{x})$ is consequence of $\sigma$. Since $v(\bar{x})=\min _{x \in A} v(x)$, there exists $\bar{\lambda} \in \Lambda$ such that $v(\bar{x})=\sum_{t \in T} \bar{\lambda}_{t} b_{t}$. Then $\sum_{t \in T} \bar{\lambda}_{t}\left(b_{t}-a_{t}(\bar{x})\right)=0$, i.e., $\bar{\lambda}_{t}=0$ if $t \notin T(\bar{x})$. Hence $v \in$ cone $\left\{a_{t}, t \in T(\bar{x})\right\}$.

In linear semi-infinite programming, cone $\left\{a_{t}, t \in T(\bar{x})\right\}$ is called the active cone at $\bar{x}$, and $v \in$ cone $\left\{a_{t}, t \in T(\bar{x})\right\}$ is the KKT optimality condition at $\bar{x}$. This condition is always sufficient for optimality and it is also necessary for LFM constraint systems ([11]).

Example 3.1 shows that uniform min-max duality does not imply uniform strong duality, even in the finite dimensional setting. It also gives an example of LFM system which fails to be FM.

Acknowledgment. The authors wish to thank the anonymous referees for their valuable suggestions, particularly those concerning Section 4.

\section{References}

[1] R.I. Boţ and G. Wanka, An alternative formulation for a new closed cone constraint qualification, Nonlinear Analysis 64 (2006) 1367-1381.

[2] R.S. Burachik and V. Jeyakumar, A dual condition for the convex subdifferential sum formula with applications, Journal of Convex Analysis 12 (2005) 279-290.

[3] R. S. Burachik and V. Jeyakumar, A new geometric condition for Fenchel's duality in infinite dimensional spaces, Mathematical Programming, Ser. B 104 (2005) 229233.

[4] N. Dinh, M.A. Goberna and M.A. López, From linear to convex systems: consistency, Farkas' lemma and applications, Journal of Convex Analysis 13 (2006) 279-290.

[5] N. Dinh, M. A. Goberna, M.A. López and T.Q. Son, New Farkas-type constraint qualifications in convex infinite programming, ESAIM: Control, Optimization and Calculus of Variations (to appear). 
[6] M.A. Goberna and M.A. López, Linear Semi-infinite Optimization, J. Wiley, Chichester (1998).

[7] R.G. Jeroslow, Uniform duality in semi-infinite convex optimization, Mathematical Programming 27 (1983) 144-154.

[8] V. Jeyakumar, The strong conical hull intersection property for convex programming, Mathematical Programming 106 (2006), 81-92.

[9] V. Jeyakumar, Characterizing set containments involving infinite convex constraints and reverse-convex constraints, SIAM Journal on Optimization 13 (2003) 947-959.

[10] V. Jeyakumar, G.M. Lee and N. Dinh, A new closed cone constraint qualification for convex optimization, Applied Mathematical Report AMR04/8, University of New South Wales, Sydney, Australia, 2004.

[11] R. Puente and V.N. Vera de Serio, Locally Farkas-Minkowski linear semi-infinite systems, TOP 7 (1999) 103-121.

[12] J. van Tiel, Convex Analysis. An Introductory Text, John Wiley \& Sons, Chichester, 1984.

[13] C. Zalinescu, Convex Analysis in General Vector Spaces, World Scientific, Singapore, 2002. 